\title{
Extracommunicative functions of language: Verbal interference causes selective categorization impairments
}

\author{
GARY LUPYAN \\ University of Pennsylvania, Philadelphia, Pennsylvania
}

\begin{abstract}
In addition to its communicative functions, language has been argued to have a variety of extracommunicative functions, as assessed by its causal involvement in putatively nonlinguistic tasks. In the present work, I argue that language may be critically involved in the ability of human adults to categorize objects on a specific dimension (e.g., color) while abstracting over other dimensions (e.g., size). This ability is frequently impaired in aphasic patients. The present work demonstrates that normal participants placed under conditions of verbal interference show a pattern of deficits strikingly similar to that of aphasic patients: impaired taxonomic categorization along perceptual dimensions, and preserved thematic categorization. A control experiment using a visuospatial-interference task failed to find this selective pattern of deficits. The present work has implications for understanding the online role of language in normal cognition and supports the claim that language is causally involved in nonverbal cognition.
\end{abstract}

What is language for? Most language research has, unsurprisingly, focused on aspects of language related to communication. However, there has long been an interest in the functions that language may have outside of communication. Such extracommunicative conceptions of language (Carruthers, 2002; Clark, 1998; Dennett, 1994; Vygotsky, 1962) have argued that in addition to its value as a communicative tool, language makes possible or facilitates certain abilities outside of the domain of communication. Support for this broad claim has come from diverse areas. Studies using infants and children have reported effects of language on object individuation (Xu, 2002), memory (Loewenstein \& Gentner, 2005), relational judgments (Kotovsky \& Gentner, 1996), and categorization (Plunkett, Hu, \& Cohen, 2008). Although the tasks used in these studies were not linguistic in nature, language had profound effects on the success of (even preverbal) infants and children.

Another source of evidence for the role of language in putatively nonlinguistic cognition comes from adults who were tested in conditions that manipulated the overtness of linguistic cues or under conditions of verbal interference. It has been found that the categorization of novel objects is improved by learning names for the categories, even when those names are entirely redundant (Lupyan, Rakison, \& McClelland, 2007). Following learning of the object-label pairing, the labels appear dynamically to facilitate visual processing of images associated with the labels (Lupyan, 2008a; Lupyan \& Spivey, 2008). The online nature of the effects of language on categorization and perception is further supported by studies showing that verbal interference reduces or eliminates this linguistic modulation (Gilbert, Regier, Kay, \& Ivry, 2006; Roberson \& Davidoff, 2000; Winawer et al., 2007). In addition to whatever long-lasting transformations that early experience with language induces on the human brain, it appears that language continues to play an online role in noncommunicative tasks, from perception and categorization to higher level processes, such as cognitive control (Emerson \& Miyake, 2003) and false-belief reasoning (Newton \& Villiers, 2007).

In addition to putting children and adults under conditions that highlight or suppress linguistic processes, another potential way to investigate the involvement of language in perception and cognition is by studying aphasic patients. Insofar as language may play a role in certain cognitive tasks, linguistic impairments may produce cognitive impairments. Indeed, observations that aphasic patients suffer deficits on a range of tasks that do not require the overt use of language have even led some to comment that one of the main functions of language is the ability to "fixate thoughts," and that "defect in language may damage thinking" (Goldstein, 1948, p. 115). The most consistent and profound nonlinguistic deficits in aphasia are observed in taxonomic categorization - specifically, tasks that require patients to selectively attend to specific stimulus dimensions. For instance, many patients are impaired at sorting objects by size or color while ignoring shape - a task requiring the abstraction of a certain color or size category

G. Lupyan, lupyan@sas.upenn.edu 
from the specific objects (Goldstein, 1948; see Noppeney \& Wallesch, 2000, for a review). After conducting and reviewing a number of such studies, Cohen and colleagues (Cohen, Kelter, \& Woll, 1980; Cohen, Woll, \& Ehrenstein, 1981) (the Konstanz Group) concluded that aphasics have a defect in the analytical isolation of single features of concepts. The authors commented that all tested subtypes of aphasic patients are "deficient if the task requires isolation, identification, and conceptual comparison of specific individual aspects of an event," but that they are equal to controls "when judgment can be based on global comparison" (Cohen et al., 1980, p. 343). Critically, this pattern is found even in patients who have demonstrably good linguistic comprehension and few semantic impairments. To illustrate, consider patient L.E.W., who had profound deficits in naming objects or actions (anomia) (Druks \& Shallice, 2000). This patient was found to be severely impaired on taxonomic-grouping tasks with even the simplest perceptual stimuli, being unable to sort colors or shapes into meaningful categories (Roberson, Davidoff, \& Braisby, 1999). However, L.E.W. demonstrated rich semantic knowledge about objects he could not name (Druks \& Shallice, 2000). Of present interest is L.E.W.'s performance on a task requiring conceptual abstraction (the odd-one-out task). In this task, the participant is presented with several pictures or words of common objects and has to select the item that does not belong, on the basis of a particular criterion. L.E.W. performed consistently with the predictions of the Konstanz Group, performing very poorly on perceptual criteria (color and size), but performing much closer to the normal baseline on arguably more difficult function judgments (e.g., choosing the pliers as not belonging to the triad nut/ pliers/wrench). These types of judgments do not involve isolating specific perceptual dimensions, but can be solved by imagining the objects used in a concrete situation - for example, using a wrench to tighten a nut. L.E.W.'s performance on the odd-one-out task stands in stark contrast with his performance on a seemingly similar task - one that requires the participant to select the correctly colored object from incorrectly colored alternatives. L.E.W. was unimpaired on this task, further demonstrating that he was not failing at object-color retrieval but at the taxonomic classification of color (Davidoff \& Roberson, 2004, Experiment 8).

Although these results are intriguing, there are numerous shortcomings to using neuropsychological findings to make conclusions about normal function. For example, it is unclear whether L.E.W.'s difficulty with taxonomic categorization was caused (in part) by his inability to name, or whether his inability to name was somehow caused by categorization difficulties (naming requires categorization). Aphasic patients differ from even the best-matched controls in a number of dimensions, only one of which is language function. The present work circumvents these limitations by adapting a task that is used with aphasic patients to normal participants who are tested under conditions of verbal interference.

\section{EXPERIMENT 1}

Experiment 1 examined whether normal participants under verbal interference show a pattern of impairments similar to that observed in aphasic patients. Specifically, it was hypothesized that performance on a task requiring the isolation of perceptual dimensions (size and color) would be compromised, whereas performance on trials requiring judgments to be made on the basis of more global comparisons would not be compromised.

To test this hypothesis, participants performed an oddone-out categorization task with and without verbal interference. Given three objects, participants had to choose the object that did not belong, on the basis of color, size, or thematic relationship (e.g., for a triad consisting of a potato, a balloon, and a cake, potato was the correct choice; balloon and cake have a common theme of party). On the basis of the findings that aphasic patients have particular difficulties with tasks requiring the isolation of perceptual features, it was predicted that verbal interference would have a stronger effect on categorization by color and size than on categorization involving broader associations (thematic relations). The design for Experiment 1 was adapted from Davidoff and Roberson's (2004, Experiment 7) study that was used with the anomic patient L.E.W., and in which he showed the predicted effect.

\section{Method}

Participants. Thirty-six undergraduates from Carnegie Mellon University and the University of Pennsylvania volunteered to participate in exchange for course credit. Participants were randomly assigned to either a picture $(n=18)$ or a word $(n=18)$ condition.

Materials. The picture condition made use of grayscale images from the modified Snodgrass and Vanderwart normalized image set (Rossion \& Pourtois, 2004). The images chosen for the color, size, and theme criterion blocks did not differ on naming times or name agreement (Rossion \& Pourtois, 2004). The word condition was identical, except that printed words were used instead of pictures. The stimulus list is presented in the Appendix. The pictures were presented in groups of three (triads); participants used a gamepad controller to select which of the three did not belong on the basis of a specific criterion. Each picture subtended approximately $5^{\circ} \times$ $7.5^{\circ}$ of visual angle.

An attempt to replicate the triads used by Davidoff and Roberson (2004, Experiment 7) with patient L.E.W. revealed that a number of the "function" triads were ambiguous, having multiple valid responses. Because it proved impossible to construct a sufficient number of unambiguous function-based triads using the normalized image set, the "function" criterion was replaced with a "theme" criterion that still required situational knowledge and global associations, but that (as pilot testing revealed) was more (though not entirely) unambiguous.

Procedure. At the start of each trial sequence, participants were instructed to select the picture (or word) that did not belong, on the basis of color, size, or theme. Examples of each type of criterion were provided, and several practice trials ensured that participants understood the nature of the task. It was stressed that color and size referred to the real-world properties of the depicted objects rather than of the pictures.

The experiment consisted of a total of 60 triads: 3 blocks (size, color, theme) $\times 2$ conditions (control, verbal interference) $\times 10$ triads/block condition. Verbal interference was instantiated through a number rehearsal task (Gilbert et al., 2006; Winawer et al., 2007). Every five trials, participants saw a prompt that informed them 


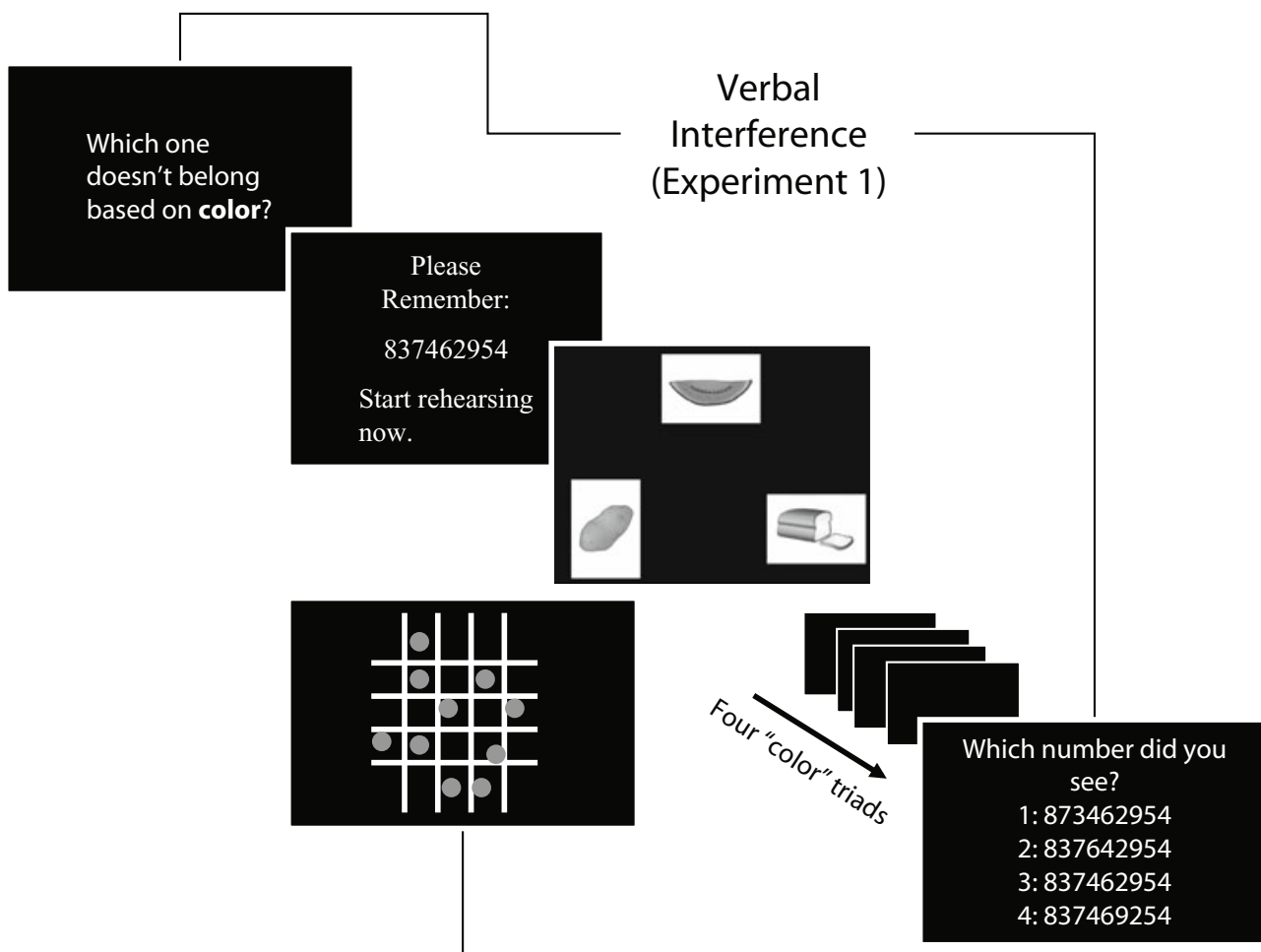

\section{Visuospatial} Interference (Experiment 2)

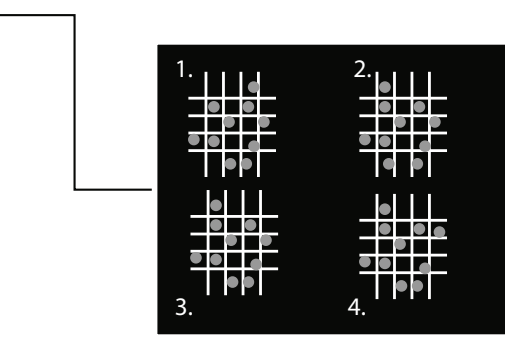

Figure 1. Experimental design illustrating verbal interference (Experiment 1) and visuospatial interference (Experiment 2). To-be-remembered digits/patterns were followed by sequences of five triads. These sequences never required switching categorization criteria in the middle of a trial block. The correct answer to the sample triad is the top picture ("watermelon").

as to whether they would have to remember a string of numbers (Figure 1). For the interference trials, the prompt was followed by a nine-digit number and an instruction to start verbally rehearsing it. The number remained on the screen for a maximum of $30 \mathrm{sec}$ (participants could initiate the trials earlier with a keypress). After completing 5 triads, participants were asked to choose the correct string in a four-alternative forced choice test. The three incorrect alternatives were generated by transposing a pair of digits. The alternatives differed only in noninitial and nonfinal digits, because pilot testing showed that participants never chose alternatives with nonmatching initial or final digits.
Verbal interference and control conditions alternated (Table 1). The starting condition and block order were counterbalanced between subjects.

\section{Results}

Mean accuracy on the verbal interference task was $M=.87(S D=.11)$ in the picture condition, and $M=$ $.83(S D=.12)$ in the word condition. Performance on the odd-one-out task was analyzed using repeated measures ANOVAs. The first analysis examined accuracy as a func-

Table 1

A Sample Trial Sequence for Experiment 1

\begin{tabular}{lcccccccccccc}
\hline & Trials & Trials & Trials & Trials & Trials & Trials & Trials & Trials & Trials & Trials & Trials & Trials \\
& $1-5$ & $6-10$ & $11-15$ & $16-20$ & $21-25$ & $26-30$ & $31-35$ & $36-40$ & $41-45$ & $46-50$ & $51-55$ & $56-60$ \\
\hline Interference condition & verbal & control & verbal & control & verbal & control & verbal & control & verbal & control & verbal & control \\
Criterion & color & color & color & color & theme & theme & theme & theme & size & size & size & size \\
\hline
\end{tabular}


tion of criterion type and interference condition. Verbal interference significantly increased errors in the picture condition from 6.8 to $14.9[F(1,17)=10.37, p=.005]$; the error increase did not reach significance for the word condition $[F(1,17)=2.60, p=.126]$; the pooled effect of verbal interference on accuracy was highly significant. Error rates without interference were comparable for all criterion types in the picture condition $[F(2,34)=1.27$, $p=.295]$. For the word condition, however, accuracy was mediated by criterion type $[F(2,34)=5.73, p=.007]$. Errors for color $(M=6.9 \%)$ and size $(M=6.1)$ were reliably lower than were those for thematic judgments $(M=$ 18.35 ), suggesting that potentially ambiguous thematic judgments are facilitated by a pictorial depiction of the objects. There were no significant interference $\times$ criterion type interactions with accuracy.

The subsequent analyses focused on reaction times (RTs). As Figure 2 (left) makes clear, error rates correlated positively with RTs, making unlikely the existence of a speed-accuracy trade-off. Incorrect responses, and responses that were shorter than $200 \mathrm{msec}$ or longer than $5,000 \mathrm{msec}$, were excluded from the analyses. Verbal interference slowed down responses for picture odd-one-out judgments by an average of $296 \mathrm{msec}[F(1,17)=14.39$, $p=.001]$, and for words by an average of $256 \mathrm{msec}$ $[F(1,17)=8.73, p=.009]$. As is shown in Figure 2 (left), the effect of verbal interference was mediated by criterion type. The prediction was that verbal interference should have an impact on perceptual judgments (size/color) more than on associative judgments (theme). This hypothesis was tested directly through a repeated measures ANOVA with interference presence and criterion type (size/color vs. thematic) as fixed factors. There was a significant interference presence $\times$ criterion interaction for picture stimuli $[F(1,17)=7.97, p=.012]$ and for word stimuli $[F(1,17)=4.96, p=.048]$. Planned tests of the effect of verbal interference on individual criterion types found that verbal interference significantly increased RTs for color $[F(1,17)=5.08, p=.038]$ and for size $[F(1,17)=11.39$, $p=.004]$, but not for theme $[F(1,17)=1.14, p=.300]$. The same pattern was observed for word stimuli [color, $F(1,17)=16.43, p<.0005$; size, $F(1,17)=4.73, p=$ .048 ; theme, $F(1,17)=0.00]$.

\section{Discussion}

Verbal interference reduced the overall accuracy and increased RTs. Of primary interest is the differential effect that verbal interference had on the blocks requiring the isolation of dimensions (color and size), in comparison with the theme block, which relied on more holistic processing of object relations. Results were largely consistent with the hypothesis that verbal interference impedes categorization, which requires isolating perceptual dimensions, as was reflected in a significant interaction between

\section{Experiment 1}

Pictures

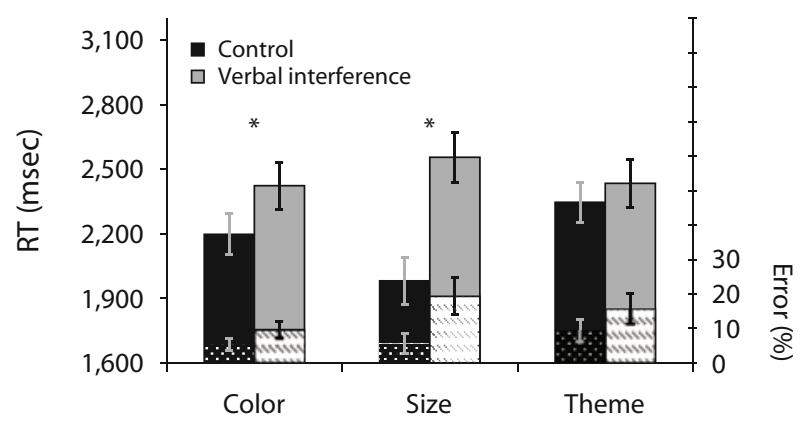

Words

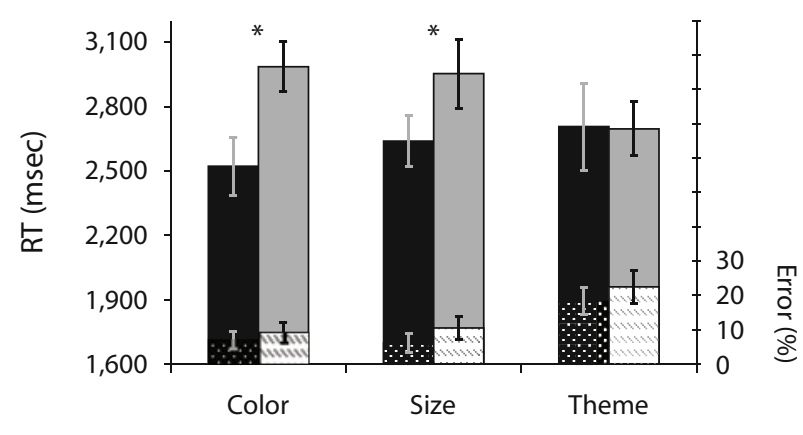

\section{Experiment 2}

Pictures

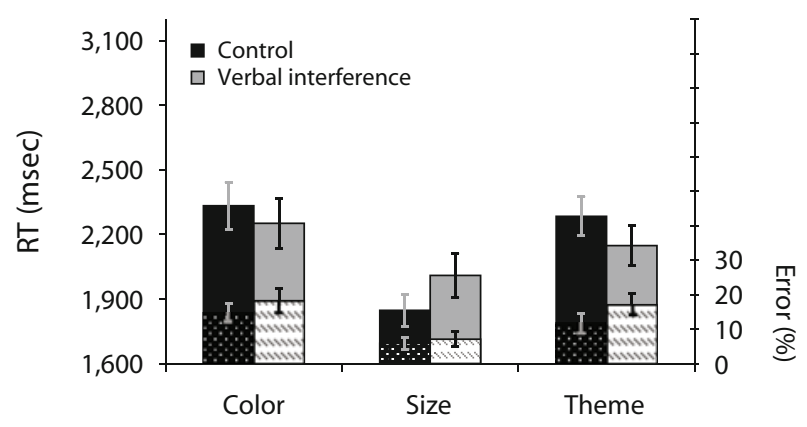

Words

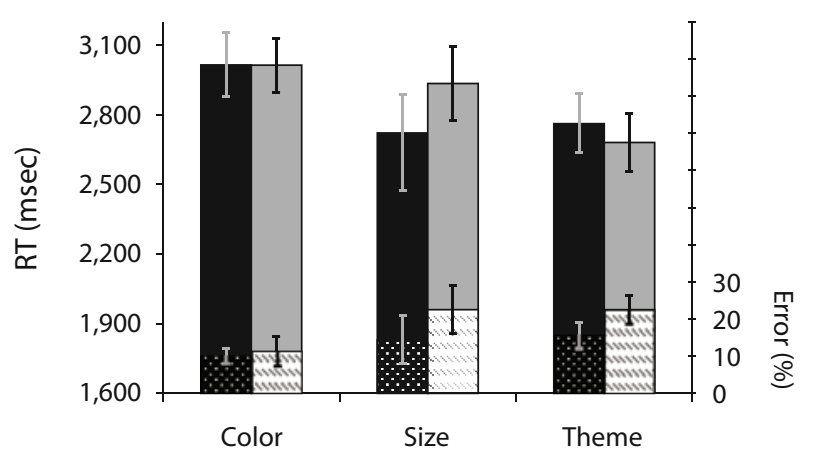

Figure 2. Left: Solid bars plot reaction times (RTs, in milliseconds) for each type of criterion with/without verbal interference. Right: RTs with/without visuospatial interference. Asterisks indicate RT differences of $p<.05$. Shaded bars plot error rates in percentages. Bars indicate standard errors of the means. 
the effect of verbal interference on color/size blocks and on the theme block.

A possibility remains that verbal interference, rather than affecting some aspect of the categorization process, interacts in some way with processing the pictures themselves. For instance, it is possible that verbal interference slows down lexical access from pictures. Insofar as lexical access is required for performing the odd-one-out task, a delay in lexical access may delay overall RTs. The word condition retained the conceptual relations while reducing the need to map from a pictorial depiction to a name. If verbal interference has its effect by slowing picture recognition (e.g., by interfering with picture naming), then it should have less or no effect on RTs when words are used as stimuli. If, however, the effect is due to the verbal interference task interfering with the "analytic decomposition" of categories (see, e.g., Cohen et al., 1980), we should see the same pattern for words as for pictures. As can be seen in Figure 2 (bottom left), the use of words as stimuli did not eliminate the differential effect of verbal interference on color and size judgments (those involving the isolation of perceptual dimensions).

\section{EXPERIMENT 2}

Experiment 1 left open the possibility that the differential effect of verbal interference on color/size versus thematic judgments was not specific to verbal interference. Although perceptual judgments were not inherently more difficult than thematic judgments (in fact, the latter had lower accuracy), it is possible that perceptual judgments are more easily disrupted by any type of interference. Experiment 2 was identical to Experiment 1, except that the verbal interference task was replaced by a visuospatial interference task. It was hypothesized that if the differential effect of verbal interference on perceptual (taxonomic) versus thematic judgments is a consequence of increasing load, then other types of interference should produce a similar pattern of results. Conversely, if the observed effect of verbal interference is more specific, demonstrating the involvement of language in the isolation of perceptual dimensions, then a nonverbal interference task should not selectively disrupt categorization performance.

\section{Method}

Participants. Thirty-three undergraduates from Carnegie Mellon University and the University of Pennsylvania participated in exchange for course credit (17 in the picture condition and 16 in the word condition). An additional 30 undergraduates (1 excluded because of high errors) were recruited to participate in a separate task that was designed to assess the relative difficulty of the two interference conditions.

Materials and Procedure. Experiment 2 was identical to Experiment 1 , except that the verbal interference task was replaced with a visuospatial interference task. At the beginning of each interference trial sequence, participants were instructed to remember the locations of nine dots that had been randomly placed on a $4 \times 4$ grid (Gilbert et al., 2006). Test trials involved choosing the correct dot pattern from a total of four alternatives. Drawing on the design of the verbal interference task, the three incorrect patterns differed from the correct pattern by a single transposition of two dots. The relative difficulty of the two interference tasks was assessed by comparing the speed and accuracy in a visual search task (as in Hermer-Vazquez, Spelke, \& Katsnelson, 1999). Participants searched for a T among Ls while simultaneously rehearsing number strings of remembering dot positions, in alternation. The target and distractors were arranged on an imaginary grid that spanned $10^{\circ}$ of visual angle; display sizes ranged from 3 to 11 elements. Both the target and distractors were presented at random orientations. Verbal and visuospatial interference alternated every six trials.

\section{Results}

To ensure that verbal and visuospatial interference were equally demanding, performance under the two interference types was measured on an unrelated task: visual search. A repeated measures ANOVA failed to find significant differences between the mean RTs between the $\operatorname{verbal}(M=829 \mathrm{msec})$ and visuospatial $(M=839 \mathrm{msec})$ interference conditions $[F(1,14)<1]$. Similarly, there was no interference condition $\times$ display size interaction $[F(2,28)=2.02, p=.15]$; the search slope during visuospatial interference was numerically greater than it was during verbal interference trials $(32 \mathrm{msec} /$ item vs. $23 \mathrm{msec} /$ item). To ensure that the lack of difference could not be attributed to floor/ceiling effects, performance was contrasted with a group of additional participants who completed the visual search task without any interference. A mixed ANOVA revealed a significant difference in RTs between the no-interference and the pooled interference conditions $[F(1,27)=4.37, p<.05]\left(M_{\text {no-interference }}=\right.$ $771 \mathrm{msec})$. There were no differences in slopes or error rates $(F \mathbf{s}<1)$. Error rates were numerically higher in conditions with slower RTs.

Mean accuracy on the visuospatial interference task for pictures and words was $.89(S D=.19)$ and $.92(S D=.15)$, respectively. These means were not significantly different from those in the verbal interference task $[F(1,68)=1.84$, $p>.18]$.

Visuospatial interference reduced overall accuracy for picture stimuli $[F(1,16)=5.04, p=.03]$ and it did so marginally for word stimuli $[F(1,15)=3.11, p=.098]$. The pooled effect was significant. There were no significant interference presence $\times$ criterion type interactions $\left(F_{\mathrm{S}}<1\right)$.

As is shown in Figure 2 (right), visuospatial interference did not significantly affect RTs for pictures or for words $\left(F_{\mathrm{S}}<1\right)$. Planned tests of the effect of visuospatial interference on RTs for individual criterion blocks failed to find any reliable effects (effect of interference on picture size judgments was marginal, $p=.092$ ).

A cross-experimental comparison of the effects of verbal versus visuospatial interference showed that verbal interference impaired performance (RTs) significantly more than did visuospatial interference: interference type $X$ presence of interference $[F(1,67)=9.44, p=.002]$. Contrary to the original prediction, the three-way interaction of criterion type $\times$ interference type $\times$ interference presence did not reach significance. However, a post hoc analysis revealed that, as was predicted, performance on color/size judgments was slowed more by verbal interference than by visuospatial interference $[F(1,67)=9.25$, $p=.003]$. 


\section{Discussion}

In comparison with verbal interference, visuospatial interference impaired performance equally (if not more) on an unrelated task (visual search), which has been used in the past to equate the difficulty of interference conditions. Although visuospatial interference increased the error rate on the picture version of the odd-one-out task, this increase was not mediated by criterion type. Analyses of RTs failed to find any reliable effects of visuospatial interference on categorization performance.

Unlike verbal interference, which selectively impaired judgments requiring the isolation of perceptual dimensions while sparing global thematic judgments, visuospatial interference produced a slight nonselective decrement in accuracy with no corresponding increase in RTs, failing to reproduce the pattern of impairments observed in aphasic patients and in the verbal interference condition of Experiment 1.

\section{GENERAL DISCUSSION}

Verbal interference selectively impaired judgments involving the isolation of perceptual dimensions (color, size), but had no significant effect on trials requiring a more global comparison, as evidenced by a significant interference condition $\times$ criterion type interaction. This effect was observed regardless of whether pictures or printed words were used as stimuli. A control experiment using a visuospatial interference task failed to find the interaction. These results provide support for the hypothesis that certain types of nonverbal categorization may depend in some way on language, even when no verbal response is required. The pattern of results in normal participants placed under verbal interference is strikingly similar to that found in aphasic patients, suggesting that language may play an online role in modulating or "sculpting" conceptual representations (Deak, 2003).

Consider that in order to group together two objects (e.g., a cherry and a brick) on the basis of their common color, imagining their color (which patients with unimpaired semantics can do) is insufficient. One has to also dynamically abstract away differences between the objects while highlighting the single dimension of color. In short: "Red cherries and red bricks may be judged to be alike mainly via what is concentrated and coined in the verbal label "red", (Koemeda-Lutz, Cohen, \& Meier, 1987, pp. 332-333).

It is important to note that although verbal interference increased RTs in normal participants, L.E.W. and many of the patients investigated by the Konstanz Group were not simply slower at performing certain types of categorization (Cohen et al., 1980; Cohen et al., 1981; Davidoff \& Roberson, 2004). Their impairments were often of a qualitative nature. For instance, when presented with an array of color swatches to be sorted into categories, rather than sorting all the swatches of one category and then moving to another, patients will often perform multiple pairwise comparisons, eventually failing at the task. They also "are meticulous to the point of pedantry and tend to be extremely concerned about slight differences in nuance that normal subjects usually overlook" (Gelb \& Goldstein, 1924, cited in De Renzi, Faglioni, Scotti, \& Spinnler, 1972, who experimentally confirmed this anecdotal account). Such performance is exactly what would be predicted if the inability to name compromises the ability to highlight a particular dimension of variation (e.g., color) while abstracting other dimensions (e.g., shape), and/or compromises the ability to focus on a particular color category (e.g., red) while abstracting over task-irrelevant within-category distinctions (Roberson et al., 1999). In fact, it has been shown that simply hearing a category label (e.g., the letter name "b") helps to deploy attention to items matching the named category, leading to faster grouping of all of the bs in a visual display (Lupyan, 2008a).

The present results provide support to extracommunicative conceptions of language. Rather than being straightforward mappings from concepts (see, e.g., Li \& Gleitman, 2002), words and the linguistic system more broadly may be critically involved in normal adult categorization. Disruptions of normal language function - whether through verbal interference or, more dramatically, through brain damage - affect not only communication, but also performance on nonverbal tasks in which language is causally involved (including, but not limited to, taxonomic categorization - the focus of the present work).

Although verbal interference tasks have recently become more popular, there is at present no comprehensive account of the mechanisms of verbal interference. The underlying assumption of the present work is that verbal interference impairs lexical representations from feeding back onto lower level (e.g., perceptual) representations (Lupyan, 2007, 2008a, 2008b; Lupyan \& Spivey, 2008). One consequence of this disruption is an impaired ability to highlight task-relevant dimensions while abstracting over task-irrelevant dimensions.

\section{AUTHOR NOTE}

Thanks to Jay McClelland, David Rakison, Jules Davidoff, and Audrey Kittredge for useful discussion. The present work was conducted while the author was at Carnegie Mellon University and the Center for the Neural Basis of Cognition, and was supported in part by an NSF Graduate Fellowship. Correspondence concerning this article should be addressed to G. Lupyan, University of Pennsylvania, 3401 Walnut St., Ste. 400A, Philadelphia, PA 19104 (e-mail: lupyan@sas.upenn.edu).

\section{REFERENCES}

CARruthers, P. (2002). The cognitive functions of language. Behavioral \& Brain Sciences, 25, 657-674.

Clark, A. (1998). Magic words: How language augments human computation. In P. Carruthers \& J. Boucher (Eds.), Language and thought: Interdisciplinary themes (pp. 162-183). Cambridge: Cambridge University Press.

Cohen, R., Kelter, S., \& Woll, G. (1980). Analytical competence and language impairment in aphasia. Brain \& Language, 10, 331-347.

Cohen, R., Woll, G., \& Ehrenstein, W. H. (1981). Recognition deficits resulting from focussed attention in aphasia. Psychological Research, 43, 391-405.

DAvidoff, J., \& Roberson, D. (2004). Preserved thematic and impaired taxonomic categorisation: A case study. Language \& Cognitive Processes, 19, 137-174.

DEAK, G. (2003). The development of cognitive flexibility and language 
abilities. In R. Kail (Ed.), Advances in child development and behavior (pp. 271-327). San Diego: Academic Press.

DenNETT, D. (1994). The role of language in intelligence. In J. Khalfa (Ed.), What is intelligence? (Darwin College Lectures). Cambridge: Cambridge University Press.

De Renzi, E., Faglioni, P., Scotti, G., \& Spinnler, H. (1972). Impairment in associating colour to form, concomitant with aphasia. Brain, 95, 293-304.

Druks, J., \& Shallice, T. (2000). Selective preservation of naming from description and the "restricted preverbal message." Brain \& Language, 72, 100-128.

Emerson, M., \& Miyake, A. (2003). The role of inner speech in task switching: A dual-task investigation. Journal of Memory \& Language, 48, 148-168.

Gilbert, A., Regier, T., Kay, P., \& Ivry, R. (2006). Whorf hypothesis is supported in the right visual field but not the left. Proceedings of the National Academy of Sciences, 103, 489-494.

Goldstein, K. (1948). Language and language disturbances. New York: Grune \& Stratton.

Hermer-Vazquez, L., Spelke, E., \& Katsnelson, A. (1999). Sources of flexibility in human cognition: Dual-task studies of space and language. Cognitive Psychology, 39, 3-36.

Koemeda-Lutz, M., Cohen, R., \& Meier, E. (1987). Organization of and access to semantic memory in aphasia. Brain \& Language, $\mathbf{3 0}$, 321-337.

Kotovsky, L., \& Gentner, D. (1996). Comparison and categorization in the development of relational similarity. Child Development, 67, 2797-2822.

Li, P., \& Gleitman, L. (2002). Turning the tables: Language and spatial reasoning. Cognition, 83, 265-294.

Loewenstein, J., \& Gentner, D. (2005). Relational language and the development of relational mapping. Cognitive Psychology, 50, 315353. doi:10.1016/j.cogpsych.2004.09.004

LuPYAN, G. (2007). The label feedback hypothesis: Linguistic influences on visual processing. Unpublished doctoral dissertation, Carnegie Mellon University, Pittsburgh, Pennsylvania.
LUPYAN, G. (2008a). The conceptual grouping effect: Categories matter (and named categories matter more). Cognition, 108, 566-577.

LuPYAN, G. (2008b). From chair to "chair": A representational shift account of object labeling effects on memory. Journal of Experimental Psychology: General, 137, 348-369.

Lupyan, G., Rakison, D., \& McClelland, J. (2007). Language is not just for talking: Labels facilitate learning of novel categories. Psychological Science, 18, 1077-1082.

Lupyan, G., \& Spivey, M. (2008). Perceptual processing is facilitated by ascribing meaning to novel stimuli. Current Biology, 18, R410-R412.

Newton, A. M., \& Villiers, J. G. D. (2007). Thinking while talking: Adults fail nonverbal false-belief reasoning. Psychological Science, 18, 574-579. doi:10.1111/j.1467-9280.2007.01942.x

NopPENEY, U., \& WALLESCH, C. (2000). Language and cognition—Kurt Goldstein's theory of semantics. Brain \& Cognition, 44, 367-386.

Plunkett, K., Hu, J., \& Cohen, L. B. (2008). Labels can override perceptual categories in early infancy. Cognition, 106, 665-681. doi:S0010-0277(07)00108-4

Roberson, D., \& DAVIDOFF, J. (2000). The categorical perception of colors and facial expressions: The effect of verbal interference. Memory \& Cognition, 28, 977-986.

Roberson, D., Davidoff, J., \& Braisby, N. (1999). Similarity and categorisation: Neuropsychological evidence for a dissociation in explicit categorisation tasks. Cognition, 71, 1-42.

Rossion, B., \& Pourtois, G. (2004). Revisiting Snodgrass and Vanderwart's object pictorial set: The role of surface detail in basic-level object recognition. Perception, 33, 217-236.

VyGotsky, L. (1962). Thought and language. Cambridge, MA: MIT Press.

Winawer, J., Witthoft, N., Frank, M., Wu, L., Wade, A., \& Boroditsky, L. (2007). Russian blues reveal effects of language on color discrimination. Proceedings of the National Academy of Sciences, 104, 7780-7785.

$\mathrm{Xu}, \mathrm{F}$. (2002). The role of language in acquiring object kind concepts in infancy. Cognition, 85, 223-250. 
APPENDIX

Stimuli Used for Size, Theme, and Color Odd-One-Out Trials

\begin{tabular}{|c|c|c|}
\hline Correct Answer & Alternative 1 & Alternative 2 \\
\hline \multicolumn{3}{|c|}{ Size } \\
\hline mountain & truck & helicopter \\
\hline windmill & kite & flag \\
\hline toaster & spoon & saltshaker \\
\hline pen & motorcycle & horse \\
\hline cap & skirt & pants \\
\hline coat & hat & crown \\
\hline tree & leaf & flower \\
\hline anchor & car & sailboat \\
\hline fish & rocking chair & donkey \\
\hline ostrich & duck & chicken \\
\hline cow & mouse & squirrel \\
\hline well & vase & bottle \\
\hline ring & sweater & shirt \\
\hline ladder & house & barn \\
\hline violin & cigar & scissors \\
\hline bee & eagle & owl \\
\hline button & blouse & coat \\
\hline pineapple & peach & onion \\
\hline cannon & caterpillar & seahorse \\
\hline rolling pin & stove & refrigerator \\
\hline \multicolumn{3}{|c|}{ Theme } \\
\hline kettle & iron & ironing board \\
\hline axe & fork & knife \\
\hline chisel & hammer & nail \\
\hline chair & lamp & light switch \\
\hline table & door & doorknob \\
\hline ball & football & football helme \\
\hline paintbrush & cigarette & ashtray \\
\hline clothespin & hair & comb \\
\hline watering can & jacket & snowman \\
\hline watch & lock & key \\
\hline shoe & hand & gloves \\
\hline glass & ruler & pencil \\
\hline potato & balloon & cake \\
\hline mitten & hanger & dress \\
\hline saw & screw & screwdriver \\
\hline whistle & plug & lightbulb \\
\hline bowl & bread & sandwich \\
\hline toothbrush & book & glasses \\
\hline cat & bird & fly \\
\hline desk & television & couch \\
\hline \multicolumn{3}{|c|}{ Color } \\
\hline pig & penguin & zebra \\
\hline deer & tiger & giraffe \\
\hline lips & ear & nose \\
\hline peacock & sheep & goat \\
\hline gorilla & camel & kangaroo \\
\hline butterfly & gun & fork \\
\hline guitar & French horn & trumpet \\
\hline eye & arm & leg \\
\hline lobster & rhinoceros & elephant \\
\hline traffic lights & barrel & fence \\
\hline corn & carrot & orange \\
\hline frying pan & wine glass & window \\
\hline watermelon & $\operatorname{dog}$ & monkey \\
\hline pumpkin & celery & asparagus \\
\hline heart & toe & thumb \\
\hline apple & swan & envelope \\
\hline cup & chain & pot \\
\hline grapes & banana & lemon \\
\hline rooster & alligator & frog \\
\hline artichoke & strawberry & tomato \\
\hline
\end{tabular}

(Manuscript received September 10, 2008; revision accepted for publication March 24, 2009.) 